\title{
Porous silicon-based rugate filters
}

\author{
Eduardo Lorenzo, Claudio J. Oton, Néstor E. Capuj, Mher Ghulinyan, \\ Daniel Navarro-Urrios, Zeno Gaburro, and Lorenzo Pavesi
}

\begin{abstract}
We report an experimental study of porous silicon-based rugate filters. We performed filter apodization, following a half-apodization approach, which successfully attenuated the sidelobes at both sides of the photonic stop band. We achieved successful reduction of interference ripples through the insertion of index-matching layers on the first and last interfaces. An apodized dielectric mirror and a rugate filter are compared: Appreciable differences in the harmonic presence and stop-band performance were observed and are commented on. Bandwidth control when index contrast is modified is also demonstrated. Finally, the possibility of combining different rugate filter designs to attain more complex responses is demonstrated by the achievement of a multi-stop-band filter. Numerical calculations for design optimization and comparison with experimental data are reported too. (c) 2005 Optical Society of America
\end{abstract}

OCIS codes: $\quad 220.4610,160.6000,350.2460,220.1230,230.4170$.

\section{Introduction}

During the past decade, porous silicon (PS) has attracted the attention of material research, not only because of its efficient visible luminescence but also because of its intriguing optical passive properties. The geometric orientation of the pores as well as their size can determine different optical properties, thus offering a wide range of applications in many fields, from optical devices to chemical sensors and biomaterials. ${ }^{1}$

Porous silicon is a dielectric mixture of silicon and air. As the structural inhomogeneities are much smaller than typical optical wavelengths, PS shows optical properties that are typical of a dielectric material with an effective refractive index $n$, which can be anisotropic. ${ }^{2}$ The value of $n$ depends directly on the parameter called porosity, which is the volumetric fraction of the pores inside the layer. More porosity leads to a lower refractive index because the dielectric effective medium contains more air.

E. Lorenzo, C. J. Oton (cjoton@ull.es), and N. E. Capuj are with the Departamento de Física Básica, University of La Laguna, Avenida Astrofísico Fco. Sánchez, E-38204, La Laguna, Tenerife, Spain. M. Ghulinyan, D. Navarro-Urrios, Z. Gaburro, and L. Pavesi are with the Istituto Nazionale per la Fisica della Materia and Dipartimento di Fisica, University of Trento, Via Sommarive 14, Povo (TN) I-38050, Italy.

Received 21 July 2004; revised manuscript received 11 February 2005; accepted 29 April 2005.

0003-6935/05/265415-07\$15.00/0

(C) 2005 Optical Society of America
PS is typically fabricated by electrochemical anodization of crystalline silicon, and the etching current density determines the porosity. ${ }^{3}$ One important advantage is that the already etched part of the layer is unaffected by subsequent current changes; thus porosity can be modulated in depth. This permits the fabrication of any refractive-index profile, so onedimensional photonic structures can be grown easily, with no need for expensive equipment. Bragg reflectors, ${ }^{4}$ microcavities,,${ }^{3,5}$ waveguides, ${ }^{6}$ and more-complex structures 7,8 made of PS have been experimentally reported. These structures can serve many applications, such as optical filters, ${ }^{9}$ resonating media for altering emission properties of luminescent substances inside the pores, ${ }^{10,11}$ and vapor sensors. ${ }^{12}$

All the multilayer devices mentioned are fabricated just by alternating the etch current between two different values: a high and a low current, which leads to layers with low and high refractive indices, respectively. However, if the current is modulated gradually, a smooth index profile can be achieved, giving rise to so-called rugate filters. These structures have been investigated for 20 years, because a smooth index profile can improve many features of optical devices with respect to dielectric multilayers. The main difficulty is their experimental achievement, which requires the gradual mixing of two different materials; rugate filters obtained by evaporation, ${ }^{13,14}$ reactive sputtering, ${ }^{15}$ plasma-enhanced chemical-vapor deposition, ${ }^{16}$ and glancing angle deposition ${ }^{17}$ were reported in the past. Birefringent rugate filters have been reported too. ${ }^{18}$ In 1997 Berger et al. reported the 
first porous silicon-based rugate filter, ${ }^{19}$ and Cunin et al. showed an application for biomolecular screening. ${ }^{20}$ Also, vacuum-evaporated PS permits a periodic smooth variation of porosity. ${ }^{21}$

In this paper, we report a detailed study of PSbased rugate filters, focusing on design, fabrication, and discussion of results. Section 2 is a theoretical introduction that describes some features of rugate filters and techniques for improving the filter response. Section 3 gives experimental details of their fabrication and characterization. Finally, in Section 4 the experimental results are shown and discussed, and they are compared with numerical calculations.

\section{Theory}

\section{A. Distributed Bragg Reflectors}

Distributed Bragg reflectors (DBRs) are photonic structures in which two dielectric media with different refractive indices $\left(n_{H}\right.$ and $\left.n_{L}\right)$ and thicknesses $\left(d_{H}\right.$ and $d_{L}$ ) are stacked alternately. The periodicity gives rise to a photonic bandgap, in which propagation of light is forbidden and the light is completely backreflected. This region is centered at $\lambda_{0}=2\left(n_{H} d_{H}\right.$ $\left.+n_{L} d_{L}\right)$. Higher harmonics appear, too, and they are located at integer multiples of the energy of the first stop band, i.e., at $\lambda_{0} / m$ for $m>1$. If the condition $n_{H} d_{H}=n_{L} d_{L}$ holds (a quarter-wave stack), the mirror shows maximum performance; for this case, only odd harmonics remain. However, very slight deviations from that condition (even less than a $1 \%$ mismatch) lead to the presence of a weak second harmonic. Therefore it is difficult to avoid a peak at $\lambda_{0} / 2$ in experimental measurements.

\section{B. Rugate Filters}

Rugate filters are structures in which the refractive index varies smoothly and periodically in depth. ${ }^{22}$ These arrangements also show bandgaps, in analogy with DBRs. These bandgaps are slightly narrower (by a factor of $\pi / 4$ ) than the bandwidth of a quarter-wave stack, but the main difference is that higher harmonics can be completely eliminated in rugate filters. To produce a stop band centered at $\lambda_{0}$ one has to use a sinusoidal index profile of the following form:

$$
n(x)=n_{0}+\frac{\Delta n}{2} \sin \left(\frac{4 \pi x}{\lambda_{0}}\right)
$$

where $x$ is the optical path, $n_{0}$ is the average of the high and low indices, and $\Delta n$ is the index contrast. However, this profile shows small-amplitude higherorder peaks. To suppress higher-order harmonics completely, one must modulate sinusoidally the logarithm of $n,{ }^{22}$ as follows:

$$
\begin{aligned}
\log [n(x)]= & \frac{\log n_{H}+\log n_{L}}{2} \\
& +\frac{\log n_{H}-\log n_{L}}{2} \sin \left(\frac{4 \pi x}{\lambda_{0}}\right) .
\end{aligned}
$$

When the index contrast is small, Eq. (2) tends to Eq. (1).

Different rugate filter designs can be combined to produce a response that, in first approximation, is the sum of single arrangements. ${ }^{22}$ For example, two different frequencies can be summed, and the resultant structure will show two bandgaps centered at the respective places. This fact is experimentally verified in Section 4 below.

\section{Apodization}

The possibility of fabricating gradual refractive-index profiles also permits the introduction of apodization functions. Apodization is a well-known technique for eliminating sidelobes coming from truncation. It involves multiplying a function by a window whose Fourier transform has small sidelobes. The trivial window is the rectangle function, which performs the truncation but introduces sidelobes related to its Fourier transform, the sinc function. These sidelobes are clearly visible at both sides of the stop band of a DBR, and they can be undesirable. If the index contrast is modulated with a smooth function, these sidelobes can be suppressed. ${ }^{19,23,24}$ There are many windows that can be used to truncate a function more smoothly: a triangle, a Gaussian, a sine, and other examples taken from signal processing theory, such as Kaiser windows. The apodization can be applied to a DBR or a rugate filter, and in both cases sidelobes can be suppressed.

\section{Index-Matching Coatings}

When the outer media (with indices $n_{\text {air }}$ and $n_{s u}$ ) are not index matched with the first and last indices of the structure, the reflectance spectrum shows ripples outside the stop band, even when the structure is apodized. These are interference fringes that arise when light bounces against the first and last interfaces. Smooth variations of refractive index can also suppress this effect by introducing index-matching layers at the boundaries. These layers are often used as broadband antireflection coatings, and linear profiles can work well, ${ }^{25}$ but the profile most employed to match indices from $n_{1}$ to $n_{2}$ is the following quintic polynomial $^{26}$ :

$$
n(t)=n_{1}+\left(n_{2}-n_{1}\right)\left(10 t^{3}-15 t^{4}+6 t^{5}\right),
$$

where $t$ is a normalized parameter proportional to the depth, which varies from 0 to 1 .

Summarizing, the higher harmonics of a DBR can be attenuated by introduction of a smooth variation of $n$, the sidelobes at both sides of the stop band are suppressed if the index profile is apodized with a smooth contrast increase and decrease, and the interference fringes can be reduced if one adds indexmatching layers that prevent reflections on the boundaries of the sample.

\section{Experiment}

We used $p^{+}$-type (100)-oriented silicon wafers ( $\rho$ $=0.01 \Omega \mathrm{cm}$ ); the electrolyte was made with a $31 \%$ 
volumetric fraction of aqueous (48 wt. \%) HF in ethanol, and the area was $1 \mathrm{~cm}^{2}$. A platinum electrode was immersed in the electrolyte, and silicon was anodized with a computer-controlled Agilent 6612C source in current source mode. A high current step $\left(450 \mathrm{~mA} / \mathrm{cm}^{2}\right.$ for $\left.0.5 \mathrm{~s}\right)$ was applied at the end of the process in free-standing samples to detach PS from the substrate. We employed a mechanical stirrer during anodization to maintain a good electrolyte exchange. ${ }^{5}$

First, to calibrate the process, we made several monolayers with different currents, covering a range from 2 to $110 \mathrm{~mA} / \mathrm{cm}^{2}$. These layers were characterized with cross-section scanning-electron microscopy analysis. From the micrographs, the thickness of the layer was measured, from which we calculated the etch rate for each current. We obtained normal reflectance spectra, in which the interference fringes were clearly observable and the optical path of the layer was measured. We then calculated the refractive index by dividing the optical by the physical thickness. The index range achievable with the currents applied was from 1.17 with $110 \mathrm{~mA}$ to 2.43 with $2 \mathrm{~mA}$. However, currents higher than $90 \mathrm{~mA}$ yielded fragile layers, and currents lower than $5 \mathrm{~mA}$ led to slow etch rates (less than $5 \mathrm{~nm} / \mathrm{s}$ ). These considerations were taken into account for the designs.

For the fabrication of rugate filters we made the assumptions that the already etched layers were unaffected by subsequent current changes and that the etching parameters were not dependent on the depth. ${ }^{5}$ However, these assumptions are not exact, as there is a slow chemical etching of the top part of the sample during anodization; also, the etching parameters drift slightly for thick samples. To prevent the occurrence of these effects, the total duration of the etching must be as short as possible. Because the etch rates are higher for higher currents, the higher the mean current that is applied, the less the total duration of the etching will be. For these reasons we have chosen $80 \mathrm{~mA}$ (a high current) as the origin of the apodization, and we have followed a half-apodization approach $^{27}$ in which the contrast is gradually increased but the maxima of the current oscillations are fixed to $80 \mathrm{~mA} / \mathrm{cm}^{2}$. This current yields a refractive index of 1.36 and is the result of a trade-off between the necessity for high etch rates and structural stability. For the same reason, the lower current limit for the apodization was fixed to $10 \mathrm{~mA}(n$ $=2.25$ ), because lower currents would have increased the total duration without a significant gain in index contrast.

We made samples both upon silicon substrates (air-PS-silicon) and free standing (air-PS-air); therefore, index matching with air and with silicon was needed to improve the performance of the filters. The current limits for these layers were less restricted because the extreme currents would affect only the initial or the last moments of the process.

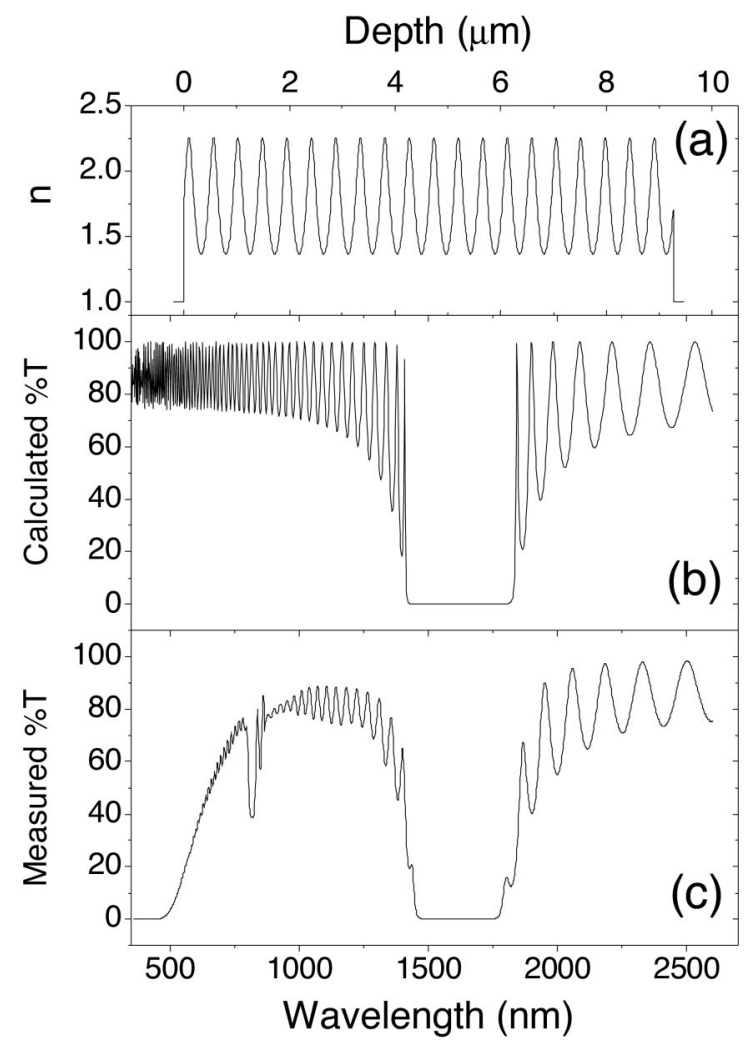

Fig. 1. Twenty-period rugate filter: (a) nominal index profile versus physical depth, (b) calculated transmission spectrum, (c) measured transmission spectrum.

Therefore we fixed them to $100 \mathrm{~mA}(n=1.22)$ and $2 \mathrm{~mA}(n=2.43)$.

The optical characterization of PS filters was carried out in a Varian Cary-5 UV-visible-near-IR spectrophotometer, with a halogen visible-near-IR lamp source. A collimated beam spot size of $1 \mathrm{~mm}$ illuminated the sample normally, and transmission and reflection spectra were obtained in a wide wavelength range $(400-2500 \mathrm{~nm})$. The overall wavelength resolution of the system was $2 \mathrm{~nm}$. However, an increase of the spot size to $15 \mathrm{~mm}$ did not yield significant changes. The aging of the samples was also characterized, because the blueshift of the spectra is a wellknown effect when fresh PS is left in ambient conditions. We observed blueshifts of $2 \%$ after 3 months. If this effect were undesirable, partial oxidation of the structures or the deposition of a protective coating would prevent it.

The filters presented here were designed for the near-IR region $1-2 \mu \mathrm{m}$. This region is important for telecommunications and for astrophysics, ${ }^{28}$ and the performance of PS-based structures is expected to be better than in the visible range, in which light absorption and scattering become considerable.

\section{Results and Discussion}

\section{A. Rugate Filter}

Figure 1 shows the results of fabrication of a 20period free-standing rugate filter. The nominal in- 


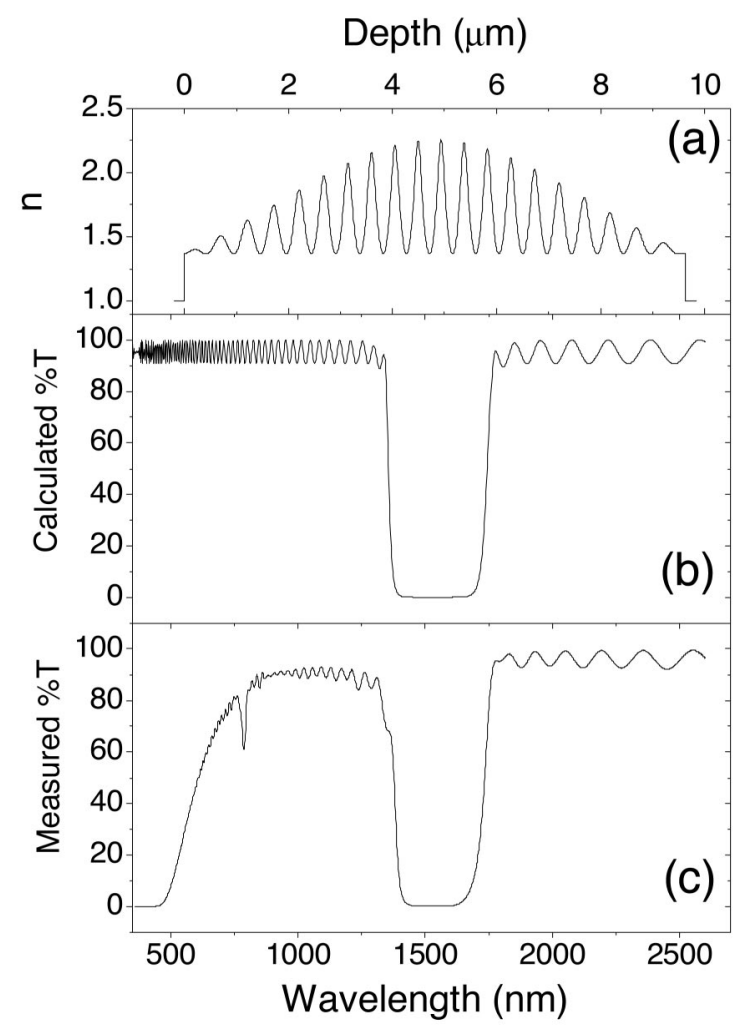

Fig. 2. Twenty-period apodized rugate filter: (a) nominal index profile versus physical depth, (b) calculated transmission spectrum, (c) measured transmission spectrum. Note the sidelobe attenuation with respect to the sample shown in Fig. 1.

dex profile is shown in Fig. 1(a). We used Eq. (2) for the calculation of the $n(x)$ curve, and we display its profile versus physical thickness. Figure 1(b) shows the calculation of the transmittance spectrum from the nominal curve. The calculation is implemented by the transfer-matrix method that is typical for multilayers. The gradual refractive-index variations were treated as discrete layers, so each oscillation was meshed into a certain number of layers until the result was independent of this number. For the sake of clarity in the interpretation, we considered neither silicon absorption (real index) nor dispersion (index not dependent on wavelength). In the visible range this is not a good approximation, but it will help the reader understand the transmission spectrum that an ideal sample would have. Figure 1(c) shows the measured transmittance spectrum, in which a stop band centered at $1600 \mathrm{~nm}$ is clearly observable. On both sides, one can see strong sidelobes in both the measured and the calculated spectra. The experiment also shows a transmission decay below $800 \mathrm{~nm}$ that is due to PS absorption, which was not taken into account in the calculations. A weak second-harmonic peak and a slightly narrower stop band are also observable in Fig. 1(c). These discrepancies are due to limitations on the assumptions made in Section 3. In spite of these small imperfections, the agreement found be-

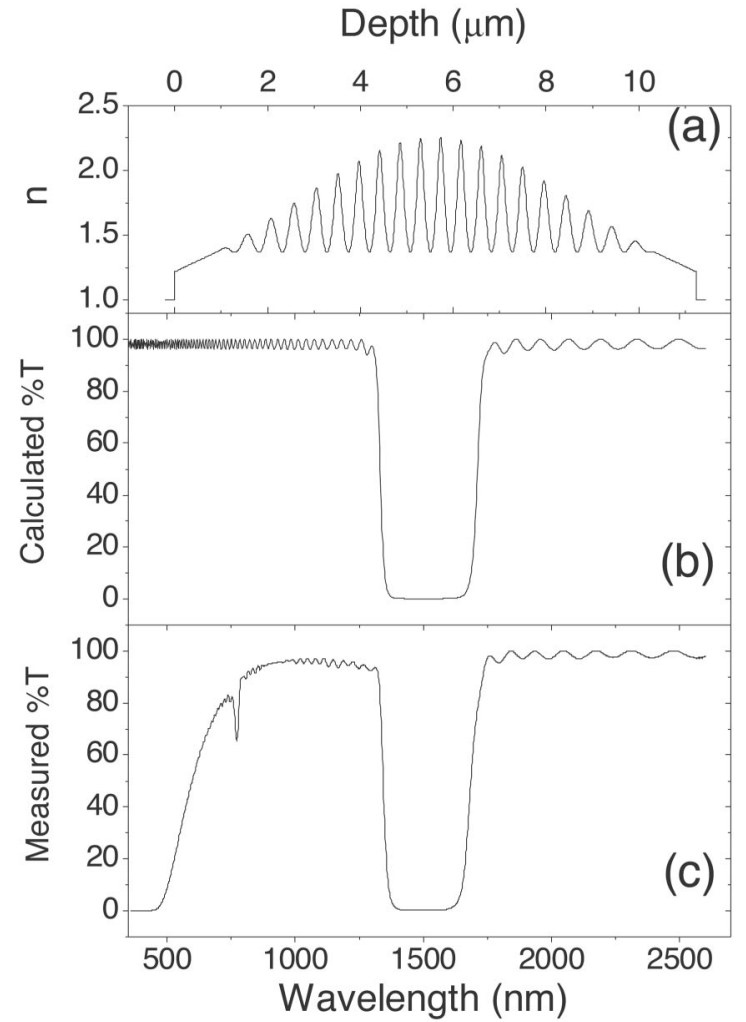

Fig. 3. Twenty-period apodized rugate filter with index-matching layers: (a) nominal index profile versus physical depth, (b) calculated transmission spectrum, (c) measured transmission spectrum. Note the absence of sidelobes and ripples.

tween the experiment and calculations demonstrates that the etching process is well controlled. The optical density (absolute value of the decimal logarithm of the transmittance) of this filter reaches a maximum of 3.4 at the center of the stop band.

\section{B. Apodization}

Figure 2 shows the same design as the previous rugate filter, but here the structure has been halfapodized. The choice of the apodization window depends on the trade-off between intensity of sidelobes and performance of the stop band. We chose $\sin (x)$ from 0 to $\pi$ because, although Kaiser, Hamming, and Gaussian windows yield weaker sidelobes, the stop band rejection level is lower and would require more oscillations. Figures 2(b) and 2(c) show the calculated and the measured spectra, respectively. When comparing these curves with Fig. 1, it is worth noting that sidelobe suppression occurs as a consequence of apodization. However, there are still ripples that oscillate at $100 \%-90 \%$ reflectance, with the intensity independent of wavelength. These ripples are not sidelobes but interference fringes that are due to slight index mismatch on the boundaries, as we show for the next sample. The optical density at the center of the stop band is 3.0, slightly lower than for the nonapodized sample, because of the wellknown fact that apodization reduces the reflectance of the stop band. 


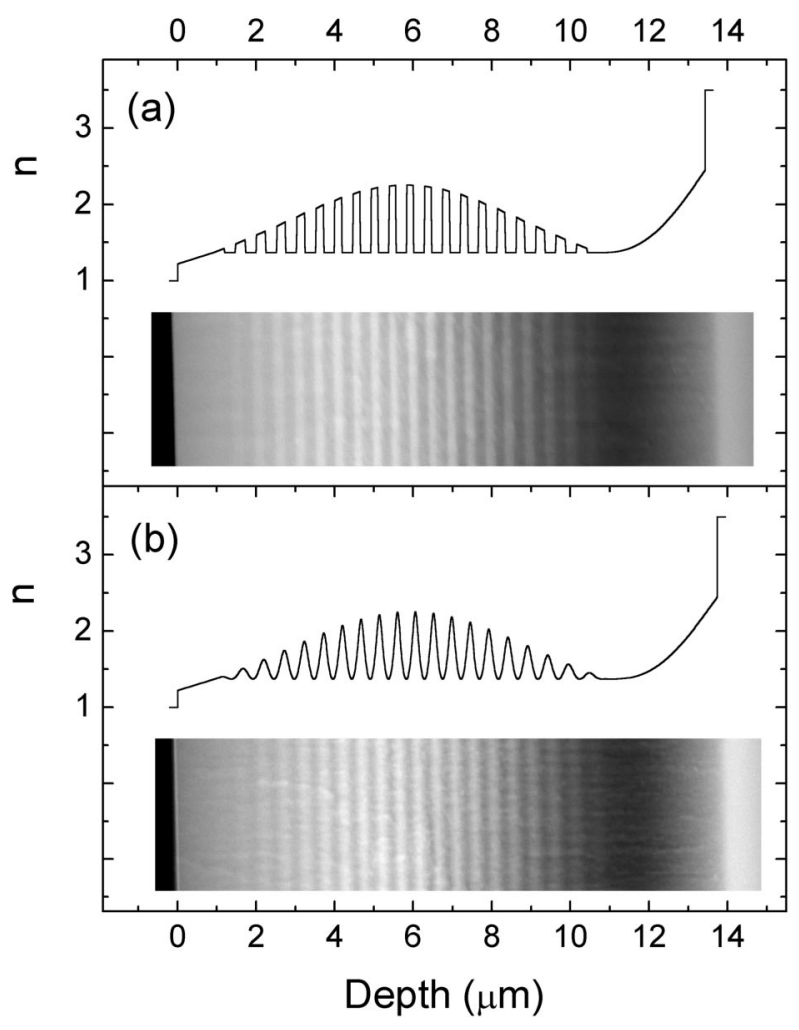

Fig. 4. Comparison of (a) an apodized DBR and (b) a rugate filter, both upon silicon substrates. The nominal index profiles of the designs are shown, and a cross-sectional scanning-electron micrograph is shown below each curve. The micrographs are scaled according to the $x$ scale shown.

\section{Index Matching}

If we add index-matching layers on the boundaries of the design described above, we obtain the structure shown in Fig. 3. As was mentioned in Section 3, we can apply currents as high as $100 \mathrm{~mA}$, because they are present only in the initial and last moments of the etching process. These layers improve the index matching because the jump becomes 1 to 1.22 instead of 1 to 1.36. This difference, with a layer with a linear profile, yields weaker interference ripples, as confirmed in Figs. 3(b) and 3(c), in which oscillations from $100 \%$ to $95 \%$ can be observed. The maximum optical density of this filter sample remains 3.0.

\section{Rugate Filter versus Distributed Bragg Reflector}

Figure 4 shows the nominal profiles of a rugate filter and of an apodized DBR, both with 20 periods and with the same contrast. The DBR was designed such that the optical thickness of all its layers remains $\lambda / 4$ (an apodized quarter-wave stack). None of the samples is free standing; thus the left-hand boundary is index matched with air (with a linear profile) and the right-hand boundary is matched with the silicon substrate, for which a half-quintic profile was designed [Eq. (3), with $t$ from 0 to 0.5 ] because a full quintic would have required too much etching time. A crosssectional scanning-electron micrograph of the samples obtained with these designs is shown below each

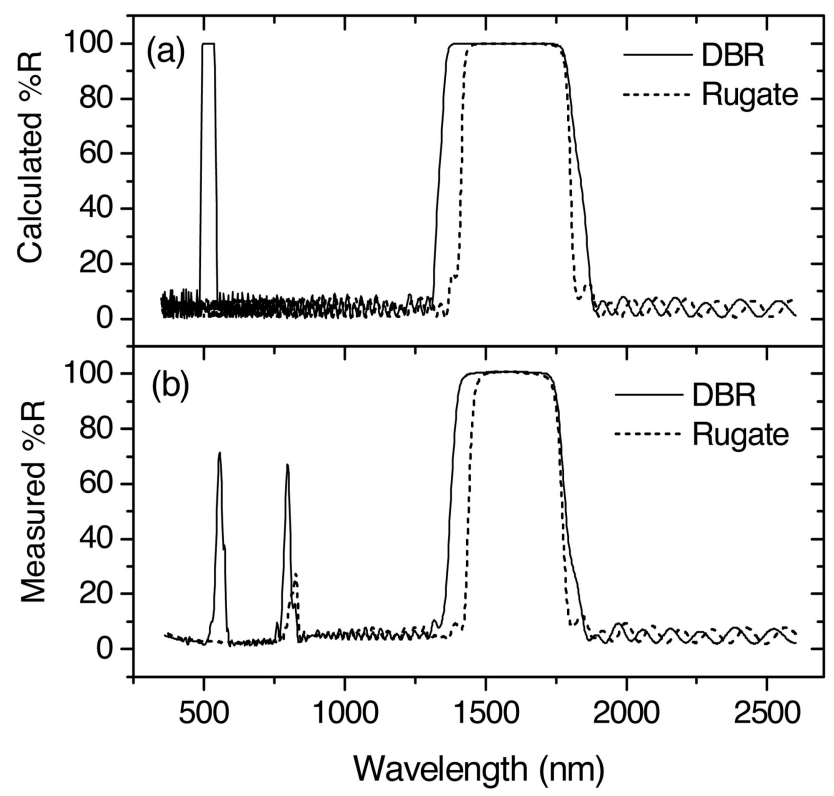

Fig. 5. (a) Calculated and (b) measured reflectance spectra of samples shown in Fig. 4.

curve. High and low porosity can be clearly distinguished as dark and bright zones, respectively. The images allow one to appreciate the abrupt index changes of the DBR with respect to the smooth variations of the rugate filter. There is also very good correspondence with the index variations of the nominal profiles, as the micrographs have been scaled according to the distances shown on the $x$ scale.

We measured the reflectance spectra of both samples, and the results are shown in Fig. 5. Figure 5(a) shows both calculated spectra, and Fig. 5(b) shows the experimental spectra. It is worth noting that in both figures the bandwidth of the rugate filter is narrower than the DBR, as predicted from theory. Besides, the sidelobes and ripples are very weak in both samples, owing to successful apodization and the presence of index-matching layers. However, the main differences observed in the calculations are the complete absence of harmonics in the rugate filter and the presence of a strong third-order harmonic in the DBR. None of the structures should show a second-harmonic peak, but the experiment shows weak peaks in both samples, as an effect of imperfection of the index profile. Nevertheless, as predicted from theory, the experimental DBR shows a strong third-harmonic peak, whereas for the rugate this harmonic is completely suppressed.

\section{E. Bandwidth Control}

The index contrast of a rugate filter or of a DBR determines the width of its stop band. To demonstrate this experimentally, we fabricated another rugate filter with less contrast. To maintain similar reflection performance, more periods are needed. Figure $6 \mathrm{com}$ pares the rugate filter shown in Fig. 3 (20 periods; contrast, 1.36-2.25; thinner curve) with a filter of reduced contrast (40 periods; contrast, 1.36-1.7; thicker 


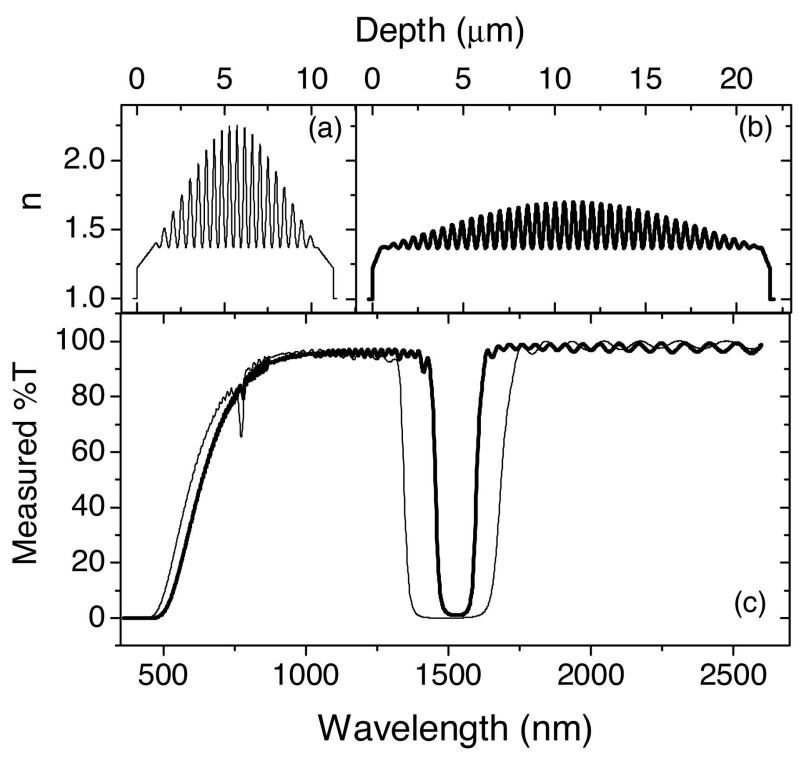

Fig. 6. Comparison of rugate filters made with different index contrasts: (a) nominal profile of a 20-period sample with 1.36 -2.25 contrast, (b) 40-period sample with $1.36-1.7$ contrast, (c) transmission spectra of the high-contrast sample (thinner curve) and the low-contrast sample (thicker curve).

curve). A bandwidth reduction from 350 to $150 \mathrm{~nm}$ can be observed. However, the narrow sample shows an optical density of 2.0 instead of 3.0.

\section{F. Superposition of Designs}

Two rugate filter designs can be combined in three ways: in parallel, in which both profiles are summed; in series, in which one is piled on the top of the other;

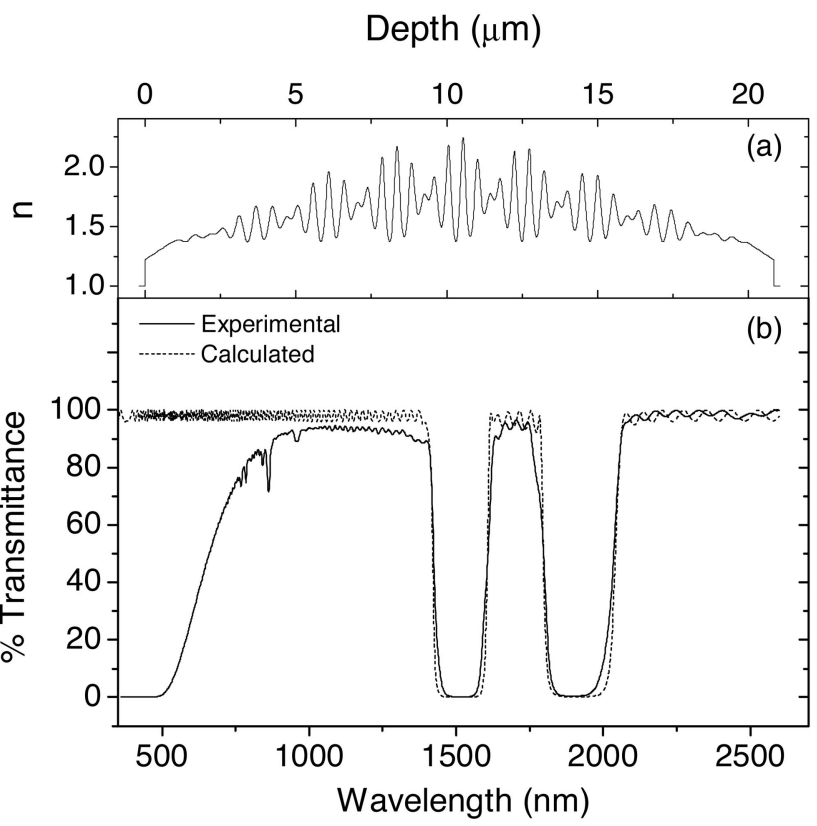

Fig. 7. Double stop-band filters centered at 1500 and $1900 \mathrm{~nm}$ with 40 and 32 periods, respectively: (a) nominal profile versus physical depth, (b) experimental and calculated transmission spectra. and shifted, ${ }^{29}$ which is midway between the other two. We present here a parallel combination of two rugate filters centered at 1500 and $1900 \mathrm{~nm}$ with 40 and 32 periods, respectively. The resultant oscillating function was apodized and index matched in the same way as the previous samples. Figure 7 shows the calculated and measured transmission spectra. A double stop band can be observed, with neither sidelobes nor ripples. The maximum optical densities measured were 3.0 at $1500 \mathrm{~nm}$ and 2.5 at $1900 \mathrm{~nm}$. The weaker reflectance of the latter is due to the smaller number of oscillation periods. The agreement between calculation and experiment for this sample confirms the possibility of combining different designs for creating multi-stop-band filters that block any desired wavelength band.

\section{Conclusions}

We have experimentally studied porous silicon-based rugate filters. We demonstrated sidelobe attenuation by means of half-apodization of the structures with a sinusoidal window. Reduction of interference ripples was experimentally observed through the insertion of index-matching layers on the boundaries of the structure. We compared an apodized distributed Bragg reflector with a rugate filter, and the main differences observed were a stronger and wider stop band for the DBR and a suppressed third-order harmonic for the rugate filter. We also studied the effect of varying the index contrast of rugate filters, in which bandwidth variations were observed. Finally, we showed an example of a parallel combination of two different designs through the fabrication of a multi-stop-band filter. The easy and cheap procedures involved in the fabrication process make these results attractive for applications in many fields, such as telecommunications, chemical and biological sensors, and astrophysics.

We thank the Instituto de Astrofisica de Canarias for offering its facilities and help for the optical characterization. We acknowledge financial support from Science and Technology Ministry of Spain Project MAT 2002-00044, Ministero dell' Instruzione, dell' Università della Ricera project IT1872, and integrated action HI2003-0180. C. J. Oton acknowledges a fellowship granted by Cajacanarias and the University of La Laguna.

\section{References}

1. M. J. Sailor, in Properties of Porous Silicon, L. T. Canham, ed. (IEE Inspec, 1997), p. 364.

2. C. J. Oton, M. Ghulinyan, Z. Gaburro, P. Bettotti, L. Pavesi, L. Pancheri, S. Gialanella, and N. E. Capuj, "Scattering rings as a tool for birefringence measurements in porous silicon," J. Appl. Phys. 94, 6334-6340 (2003).

3. L. Pavesi, "Porous silicon dielectric multilayers and microcavities," Riv. Nuovo Cim. 20, 1-76 (1997).

4. V. Agarwal and J. A. del Rio, "Tailoring the photonic band gap of a porous silicon dielectric mirror," Appl. Phys. Lett. 82, 1512-1514 (2003).

5. M. Ghulinyan, C. J. Oton, G. Bonetti, Z. Gaburro, and L. 
Pavesi, "Free-standing porous silicon single and multiple cavities," J. Appl. Phys. 93, 9724-9729 (2003).

6. P. Ferrand, R. Romestain, and J. C. Vial, "Photonic band-gap properties of a porous silicon periodic planar waveguide," Phys. Rev. B 63, 115106 (2001).

7. L. Dal Negro, C. J. Oton, Z. Gaburro, L. Pavesi, P. Johnson, A. Lagendijk, R. Righini, M. Colocci, and D. S. Wiersma, "Light transport through the band-edge states of Fibonacci quasicrystals," Phys. Rev. Lett. 90, 055501 (2003).

8. R. Sapienza, P. Costantino, D. Wiersma, M. Ghulinyan, C. J. Oton, and L. Pavesi, "Optical analogue of electronic Bloch oscillations,” Phys. Rev. Lett. 91, 263902 (2003).

9. C. Mazzoleni and L. Pavesi, "Application to optical components of dielectric porous silicon multilayers," Appl. Phys. Lett. 67, 2983-2985 (1995)

10. L. Pavesi, C. Mazzoleni, A. Tredicucci, and V. Pellegrini, "Controlled photon emission in porous silicon microcavities," Appl. Phys. Lett. 67, 3280-3282 (1995).

11. Y. Zhou, P. A. Snow, and P. St. J. Russell, "Strong modification of photoluminescence in erbium-doped porous silicon microcavities," Appl. Phys. Lett. 77, 2440-2442 (2000).

12. P. Allcock and P. A. Snow, "Time-resolved sensing of organic vapors in low modulating porous silicon dielectric mirrors," J. Appl. Phys. 90, 5052-5057 (2001).

13. C. S. Bartholomew, M. D. Morrow, H. T. Betz, J. L. Grieser, R. A. Spence, and N. P. Murarka, "Rugate filters by laser flash evaporation of $\mathrm{SiO}_{x} N_{y}$ on room-temperature polycarbonate," J. Vac. Sci. Technol. A 6, 1703-1707 (1988).

14. W. J. Gunning, R. L. Hall, F. J. Woodberry, W. H. Southwell, and N. S. Gluck, "Codeposition of continuous composition rugate filters,” Appl. Opt. 28, 2945-2948 (1989).

15. A. F. Jankowski, L. R. Schrawyer, and P. L. Perry, "Reactive sputtering of molybdenum-oxide gradient-index filters," J. Vac. Sci. Technol. A 9, 1184-1187 (1991).

16. P. L. Swart, P. V. Bulkin, and B. M. Lacquet, "Rugate filter manufacturing by electron cyclotron resonance plasmaenhanced chemical vapor deposition of $\operatorname{SiN}_{x}$, Opt. Eng. 36, 1214-1219 (1997).
17. K. Kaminska, T. Brown, G. Beydaghyan, and K. Robbie, "Rugate filters grown by glancing angle deposition," in Applications of Photonic Technology 5, R. A. Lessard, G. A. Lampropoulos, and G. W. Schini, eds., Proc. SPIE 4833, 633-639 (2003).

18. A. J. McPhun, Q. H. Wu, and I. J. Hodgkinson, "Birefringent rugate filters," Electron. Lett. 34, 360-361 (1998).

19. M. G. Berger, R. Arens-Fischer, M. Thönissen, M. Krüger, S. Billat, H. Lüth, S. Hilbrich, W. Theiss, and P. Grosse, "Dielectric filters made of PS: advanced performance by oxidation and new layer structures," Thin Solid Films 297, 237240 (1997)

20. F. Cunin, T. A. Schmedake, J. R. Link, Y. Li, J. Koh, S. Bhatia, and M. Sailor, "Biomolecular screening with encoded poroussilicon photonic crystals," Nature Mater. 1, 39-41 (2002).

21. K. Kaminska, T. Brown, G. Beydaghyan, and K. Robbie, "Vacuum evaporated porous silicon photonic interference filters," Appl. Opt. 42, 4212-4219 (2003).

22. B. G. Bovard, "Rugate filter theory: an overview," Appl. Opt. 32, 5427-5442 (1993).

23. W. H. Southwell and R. L. Hall, "Rugate filter sidelobe suppression using quintic and rugated quintic matching layers," Appl. Opt. 28, 2949-2951 (1989).

24. W. H. Southwell, "Using apodization functions to reduce sidelobes in rugate filters," Appl. Opt. 28, 5091-5094 (1989).

25. C. C. Striemer and P. M. Fauchet, "Dynamic etching of silicon for broadband antireflection applications," Appl. Phys. Lett. 81, 2980-2982 (2002).

26. W. H. Southwell, "Gradient-index antireflection coatings," Opt. Lett. 8, 584-586 (1983).

27. H. A. Abu-Safia, A. I. Al-Sharif, and I. O. Abu Aljarayesh, "Rugate filter sidelobe suppression using half-apodization," Appl. Opt. 32, 4831-4835 (1993).

28. A. R. Offer and J. Bland-Hawthorn, "Rugate filters for $\mathrm{OH}-$ suppressed imaging," in Optical Astronomical Instrumentation, S. D’Odorics, ed., Proc. SPIE 3355, 970-978 (1998).

29. W. H. Southwell, "Extended-bandwidth reflector designs by using wavelets," Appl. Opt. 36, 314-318 (1997). 